\title{
Colaboração em Ambientes Educacionais com Realidade Aumentada
}

\author{
Oliveira, F.C. Pós Graduação/Universidade Metodista de Piracicaba (Unimep) \\ francisco.o@hotmail.com
}

Kirner, C. Centro de Educação Aberta e à Distância/Universidade Federal de Ouro Preto ckirner@cead.ufop.br

Forte, C. Depto de Computação/Anhanguera Educacional (FAC SBO) cleberforte@hotmail.com Santin, R. Depto de Computação/Universidade Federal dos Vales do Jequitinhonha e Mucuri rasantin@hotmail.com

\begin{abstract}
Collaborative Learning Environments with Augmented Reality
Abstract. Collaboration is an inherent activity in he teaching/learning process, having worth at professional actions. However, the computer support used to implement and improve collaboration is too restricted. This paper presents new ways to use computer to support collaboration, emphazing tangible interactions complemented with images and sounds. An augmented reality tool named SACRA was used in this way to produce collaborative applications full of tangible interactions, images and sounds, that were tested with local and remote users.
\end{abstract}

Keywords - augmented reality, collaboration, learning environments

Resumo. A colaboração é uma atividade inerente ao processo de ensino/aprendizagem, muito valorizada também nas ações profissionais. No entanto, o apoio computacional, para a implementação e potencialização da colaboração, ainda tem sido usado de maneira restrita. Este trabalho apresenta novas formas de uso do computador em atividades colaborativas, procurando explorar interações tangíveis complementadas com imagens e sons. Uma ferramenta de autoria com realidade aumentada, denominada SACRA foi usada, nesse sentido, para o desenvolvimento de aplicações colaborativas locais e à distância.

Palavras-chave - ambientes educacionais, colaboração, realidade aumentada.

\section{Introdução}

A evolução tecnológica, ocorrida nas últimas décadas, vem influenciando fortemente as técnicas pedagógicas das várias modalidades de ensino/aprendizagem, incluindo ensino presencial, semi-presencial e à distância. Atualmente, a maioria dessas modalidades ainda é presencial, onde educadores e educandos ocupam o mesmo espaço físico e atuam no mesmo instante; é o método tradicional de ensino que já perdura por muitos séculos. Outra forma de educar é a modalidade não presencial (à distância) que, embora não seja tão antiga quanto a primeira, tampouco é tão recente como se poderia pensar. No Brasil, um dos primeiros métodos não presenciais utilizados foi o ensino através de correspondências, oferecido pelo Instituto Universal Brasileiro desde 1941 (2008). Em alguns países da Europa, como Inglaterra e Alemanha, esse tipo de ensino é ainda mais antigo, tendo surgido no século XIX. 
Entretanto, a colaboração, que é um dos aspectos importantes no processo de ensino/aprendizagem, não é explorada no ensino por correspondência, pelas dificuldades inerentes. A colaboração, em ambientes educacionais, é importante, na medida em que baseia-se em trabalhos em grupo, melhorando as condições de aprendizagem e replicando várias situações dos ambientes de Educação à Distância (EaD). Por esse motivo, a colaboração vem sendo foco de grande interesse pela comunidade educacional (Smith, 1992). Dentre as vantagens, observadas em processos colaborativos, estão a competição e a cooperação que surgem naturalmente entre os membros do grupo, ao longo do desenvolvimento, possibilitando melhores resultados do que quando o processo se dá de forma isolada (Kirner 2005).

Este artigo descreve o uso da Realidade Aumentada (RA) como tecnologia de apoio para a colaboração em processos educacionais. São apresentados alguns exemplos básicos de aplicações colaborativas locais e remotas com RA, envolvendo Educação em ambientes presenciais e à distância.

\section{Realidade Aumentada}

\subsection{Definições e Considerações}

Conforme Azuma (1997), Realidade Aumentada é uma tecnologia que permite ao usuário interagir com objetos tridimensionais inseridos no mundo real, ou seja, o ambiente físico em que vivemos. Os objetos tridimensionais (3D) gerados por computador, também conhecidos como objetos virtuais, apresentam vantagens em relação às imagens tradicionais, na medida em que permitem maior interação, já que esses objetos podem ser manipulados pelo usuário com vários pontos de vista. Esses objetos podem ser gerados diretamente num editor de texto, exigindo conhecimentos de programação. No entanto, existem diversas ferramentas, inclusive gratuitas, que geram os objetos de forma mais rápida, utilizando boas interfaces gráficas. Um desses softwares é o Vivaty Studio (2008).

Bajura (1995) define RA como sendo a sobreposição de objetos virtuais no mundo real, mediante algum dispositivo tecnológico, incrementando, dessa forma, a visão do usuário.

Azuma (2001) descreve quatro maneiras de se aplicar a RA. O que as diferenciam é o tipo de display utilizado. As quatro modalidades de RA são: sistema de visão ótica direta; sistema de visão direta por vídeo; sistema de visão por vídeo baseado em monitor e sistema de visão ótica por projeção. Como as aplicações que serão mostradas no trabalho são exclusivas de sistemas baseado em monitor (Figura 1) não serão tratados os demais tipos. 


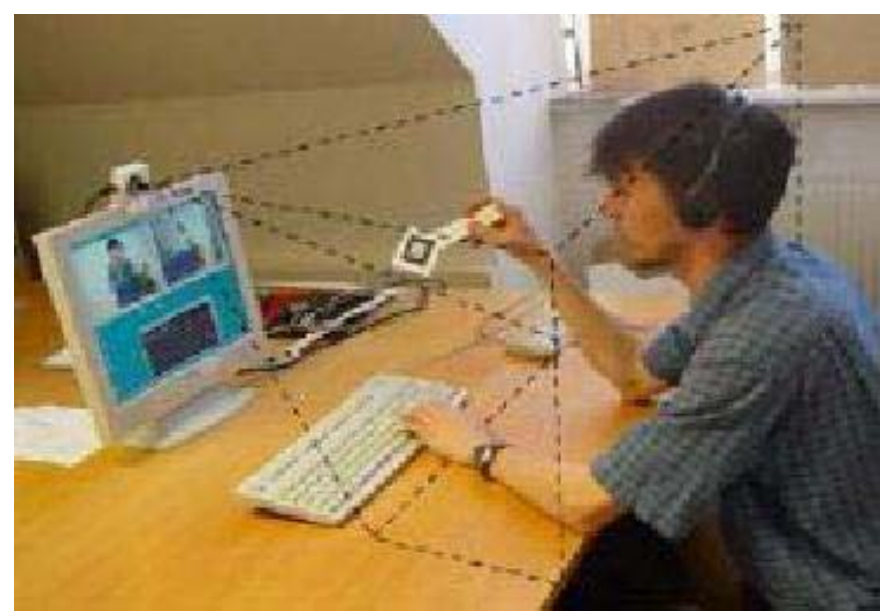

Figura 1 - Sistema de RA baseado em monitor (Barakonyi, 2003)

O sistema baseado em visão através de vídeo utiliza uma webcam para capturar a cena real. Uma vez que a cena real é capturada, seus elementos reais são misturados com objetos virtuais gerados por computador, antes de ser mostrada em um monitor (Barakonyi, 2003), conforme Figura 1.

\subsection{Hardaware e Software}

Para sua aplicação, a RA requer tanto recursos de hardware, quanto de software. Uma aplicação básica de RA necessita de um computador comum, além de um dispostivo de captura de imagem, que pode ser uma webcam igualmente comum. Quanto ao software, um dos mais utilizados é o ARToolKit (2008), inicialmente desenvolvido pela Universidade de Osaka pelo Dr Hirokazu Kato. Trata-se de um programa livre e de código aberto, o que equivale a dizer que pode ser alterado conforme a necessidade de cada uso. Essa ferramenta tem por função rastrear e posicionar, em tempo real, os objetos $3 \mathrm{D}$ em relação à câmera, através de uma forma padrão, geralmente constituída por um marcador (pedaço de papel com uma moldura desenhada e tendo no seu interior um símbolo qualquer). Essa capacidade de rastreamento da posição do marcador em tempo real, é baseada numa biblioteca de programação que utiliza recursos de visão computacional para alinhar objetos virtuais com seus respectivos padrões de marcadores. A Figura 2 ilustra uma aplicação básica de RA proposto por Zhou (2005). A figura ilustra os marcadores representados por impressões quadrangulares no livro mostrado; é para esses marcadores que são "trazidos" os objetos virtuais, que nesse caso estão representados por bonecos brancos e verdes.
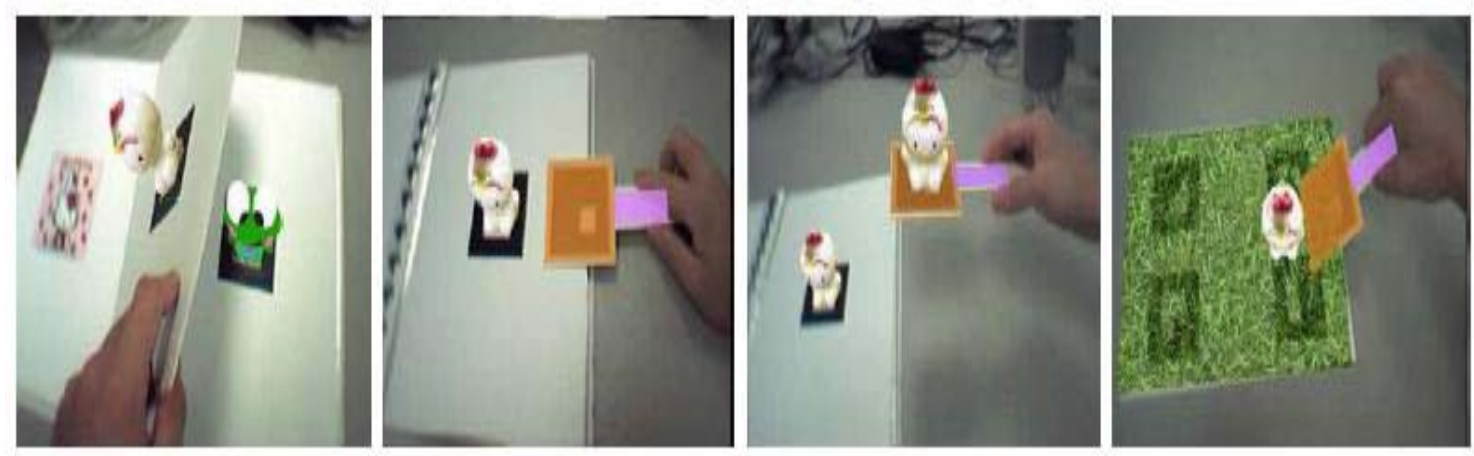

Figura 2 - Ambiente baseado em RA (Zhou, 2005) 


\section{Colaboração Local e Remota}

Em sistemas computacionais, a colaboração local ocorre quando um ou mais usuários cooperam e atuam no mesmo ambiente. A Figura 3 mostra dois exemplos desse tipo de ambiente. O primeiro caso (a) ilustra dois usuários interagindo no mesmo ambiente e no mesmo elemento visual (Agrawala, 1997). No segundo exemplo (b), o mesmo ambiente pode ser visualizado e manipulado pelos dois usuários, com a diferença de que, nesse caso, é necessário o uso de capacetes especiais, chamados HMD (Head Mounted Display) que possuem câmeras acopladas apontando na mesma direção da visão (Billinghurst, 2002).

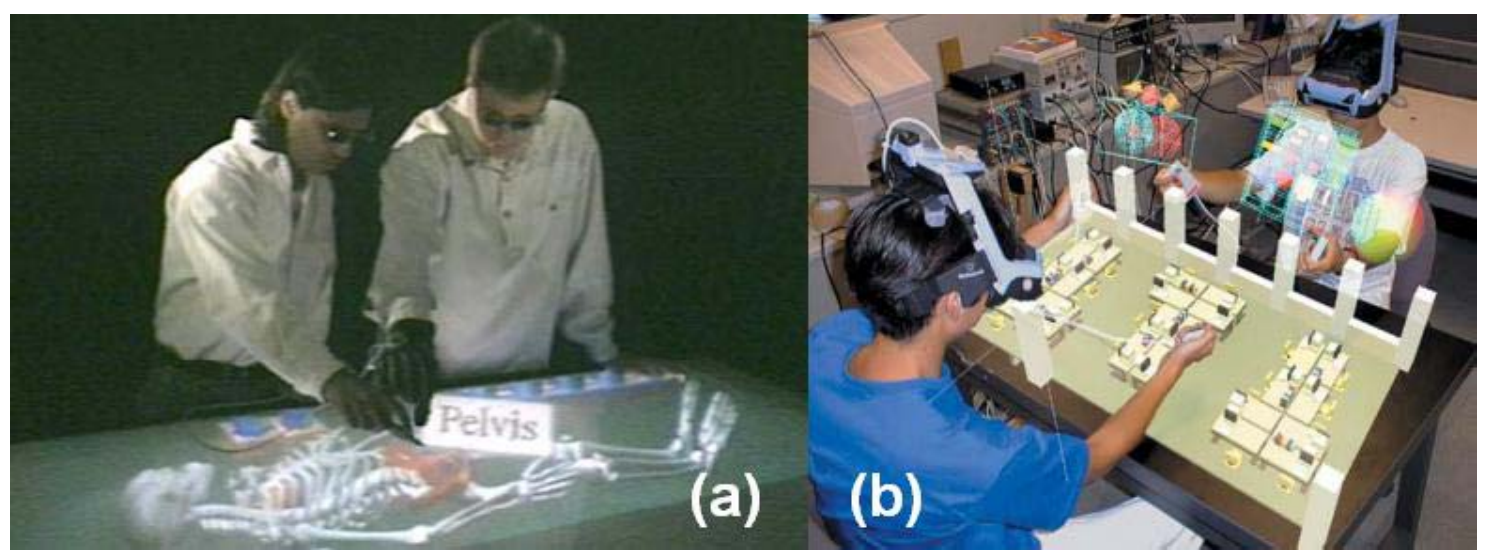

Figura 3 - Exemplos de ambientes colaborativos com RA, (Billinghurst, 2002)

A colaboração remota, por sua vez, ocorre quando os participantes estão separados fisicamente. Existem duas modalidades desse tipo de colaboração: síncrona e assíncrona. A diferença entre ambas situações está na presença simultânea ou não dos usuários envolvidos. No caso da colaboração síncrona, a aplicação ocorre ao mesmo tempo para todos os usuários, porém em locais diferentes. Já o tipo assíncrono ocorre em tempos diferentes.

\section{Aplicações com RA}

\subsection{Studierstube}

Desenvolvido pela Universidade de Tecnologia de Viena, Studierstube (Schmalstieg, 2002) é uma ferramenta para o desenvolvimento de aplicações com RA, assim como o ARTooKit. O sistema permite a geração de imagens tridimensionais que podem ser compartilhadas tanto face a face, quanto de forma remota. A Figura 4 ilustra a aplicação do Construct3D, uma ferramenta projetada para construir figuras tridimensionais em ambientes baseados em RA sendo desenhada especificamente para aplicações educacionais de matemática e geometria. A grande vantagem desse sistema é que o estudante consegue ver as imagens das figuras geométricas como objetos 3D, aumentado sua percepção na elaboração desses objetos geométricos. 

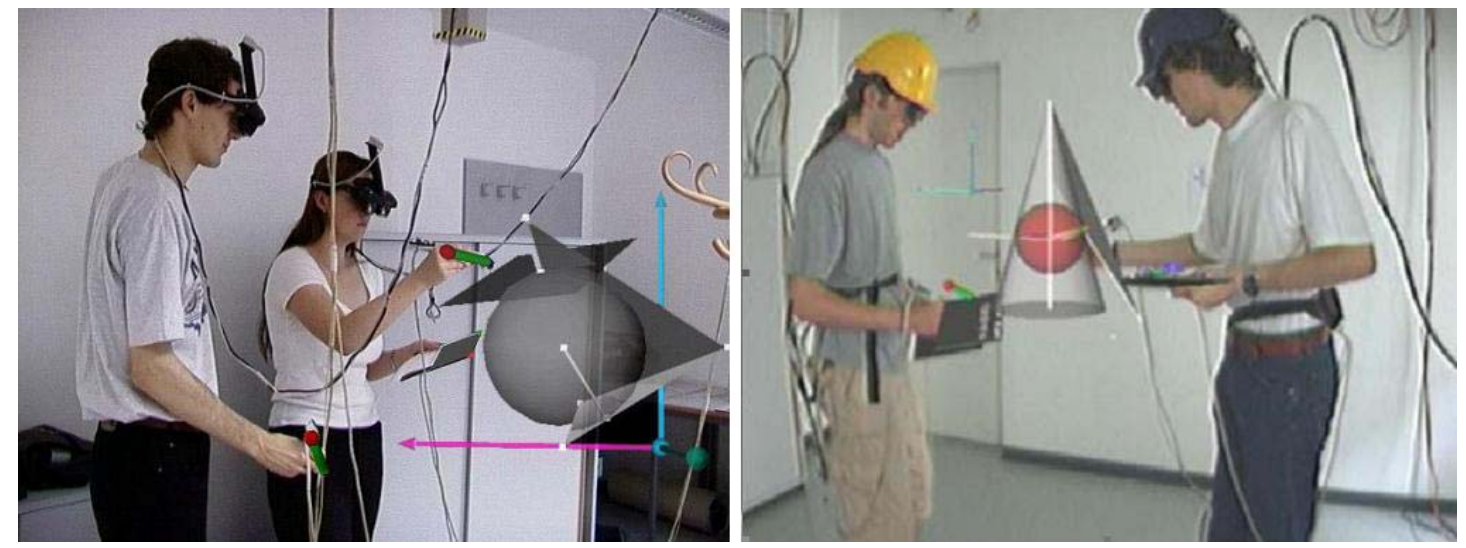

Figura 4 - Estudantes operando o Construct3D (Schmalstieg, 2002)

\subsection{Sistema SACRA}

O Sistema de Autoria Colaborativa com Realidade Aumentada (SACRA) é uma ferramenta de autoria desenvolvida para usuários de computadores que não dominam conhecimentos de programação (Santin, 2008). O SACRA tem por objetivo dar ao usuário a liberdade suficiente para que possa desenvolver suas próprias aplicações de RA, sem depender de outros profissionais. Assim como a grande maioria das ferramentas de autoria, o sistema SACRA foi elaborado com uso de interfaces gráficas, às quais são mais estimulantes para o usuário, na medida que reforçam sua percepção. A utilização de interfaces tangíveis, de acordo com Santin (2008), possibilita a potencialização da percepção dos usuários, visto que utilizam a intuição natural na manipulação de objetos virtuais no mundo real, que é justamente o ambiente em que o usuário está mais acostumado a trabalhar.

\subsubsection{Ambiente do Sistema SACRA}

O ambiente de RA tangível oferecido pelo sistema SACRA permite a autoria de mundos virtuais usando colaboração face a face e remota. O desenvolvimento do sistema foi baseado nas versões 2.65 e 2.72 .1 da biblioteca ARToolKit (2008), uma vez que esta utiliza dispositivos de baixo custo, envolvendo computador com webcam. A colaboração remota depende de conexões em rede suportada por outro software desenvolvido e incorporado no sistema, consistindo do NetARToolKit (Lucas, 2008). As propriedades, visibilidade, posição e orientação dos marcadores do SACRA, permitem aos seus usuários interagirem de forma colaborativa com o sistema, produzindo ambientes interativos com objetos reais e virtuais misturados, complementados com imagens e sons ativados nas interações.

Utilizando-se de técnicas de cooperação, coordenação e comunicação, a autoria também possibilita que usuários remotos compartilhem o mesmo ambiente na construção de mundos virtuais; a Figura 7 apresenta um exemplo de colaboração entre dois usuários remotos.

\subsubsection{Funcionalidades do SACRA}

Dois tipos de marcadores foram implementados no SACRA; os que têm funções pré-definidas e aqueles que podem ser cadastrados a qualquer momento pelo usuário. Os primeiros representam as funções que foram implementadas na estrutura do ARToolKit e estão inseridos no diretório "Data/vrml_data.dat" do sistema. A Figura 5 mostra parte da estrutura desse arquivo, descrevendo as oito funções "define". 


\begin{tabular}{|l|ll|}
\hline \#define INSPECTOR & 0 \\
Arquivo & \#define CONTROL & 1 \\
\#ata/vrml_data.dat & \#define COPY & 2 \\
\#define TRANSPORT & 3 \\
& \#define ERASER & 4 \\
\#define STATUS & 5 \\
& \#define PATH & 6 \\
\#define LOCK & 7 \\
\hline
\end{tabular}

Figura 5 - Definição das funcionalidades dos marcadores

Os marcadores do segundo tipo representam aqueles que podem ser cadastrados, a partir do oitavo marcador (\#define LOCK 7) observado no arquivo ".dat" exemplificado na Figura 5. Eles assumem o papel de marcadores de referência, denominados REF. A referência é um marcador que recebe um conjunto de pontos e objetos virtuais, constituindo uma aplicação ou parte dela, permitindo assim que os usuários interajam com os pontos e objetos virtuais presentes no espaço cadastrado no sistema. Cada REF pode ter diversos pontos, que referenciam um ou mais objetos virtuais. A Figura 6 descreve um ambiente baseado em RA com as oito placas de funcionalidades do SACRA; mostra também simulações de objetos virtuais e uma placa REF.

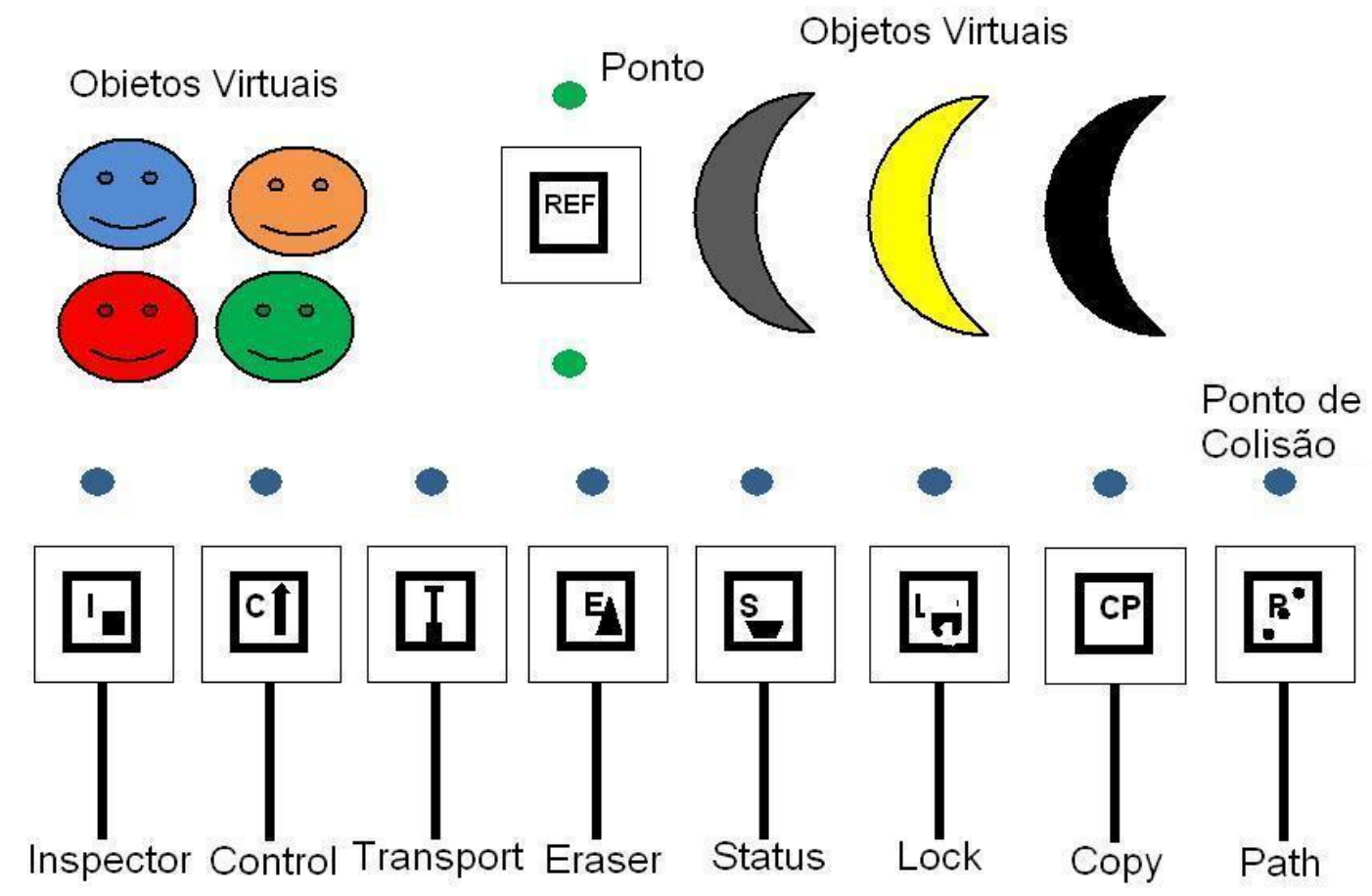

Figura 6 - Exemplos de marcadores de ações e referência (Santin, 2008)

A função de cada placa (marcador) é definida na Tabela 1. Os marcadores de ações atuam nos pontos associados às referências através do ponto de colisão, que pode 
ou não coincidir com o centro do marcador; na figura acima, esses pontos (azuis) foram deslocados.

Tabela 1. Lista das placas de ação com suas respectivas funções.

\begin{tabular}{|l|l|}
\hline \multicolumn{1}{|c|}{ PLACA } & \multicolumn{1}{c|}{ FUNÇÕES } \\
\hline Inspector & Inspecionar ou recadastrar novos pontos \\
\hline Control & fazer a troca dos objetos virtuais no ambiente \\
\hline Copy & fazer cópias de objetos virtuais do cenário \\
\hline Transport & reposicionar o ponto em novas orientações \\
\hline Eraser & excluir os pontos e desalocar os objetos virtuais da memória \\
\hline Status & mostrar ao usuário os valores das principais variáveis \\
\hline Path & realizar percursos entre as REFs mediante rastros (Figura 7) \\
\hline Lock & permite o bloqueio e desbloqueio das operações remotas \\
\hline
\end{tabular}

Para permitir a atuação dos usuários de forma remota e colaborativa, foi criado um ambiente virtual comum a vários usuários (Santin 2008), conforme ilustra a Figura 7 , onde se observa a visão de dois usuários. A parte esquerda da Figura (a) mostra o percurso realizado pelo usuário 1, o qual deverá ser percorrido pelo usuário 2 na Figura (b); o trajeto é representado na figura por pequenas esferas azuis. Esse processo demonstrou que, enquanto o usuário 1 traça o caminho a ser percorrido, o segundo, remotamente posicionado, observa atentamente com o propósito de, num segundo momento, tentar realizar o transporte da peça do helicóptero para encaixá-la em seu devido lugar.
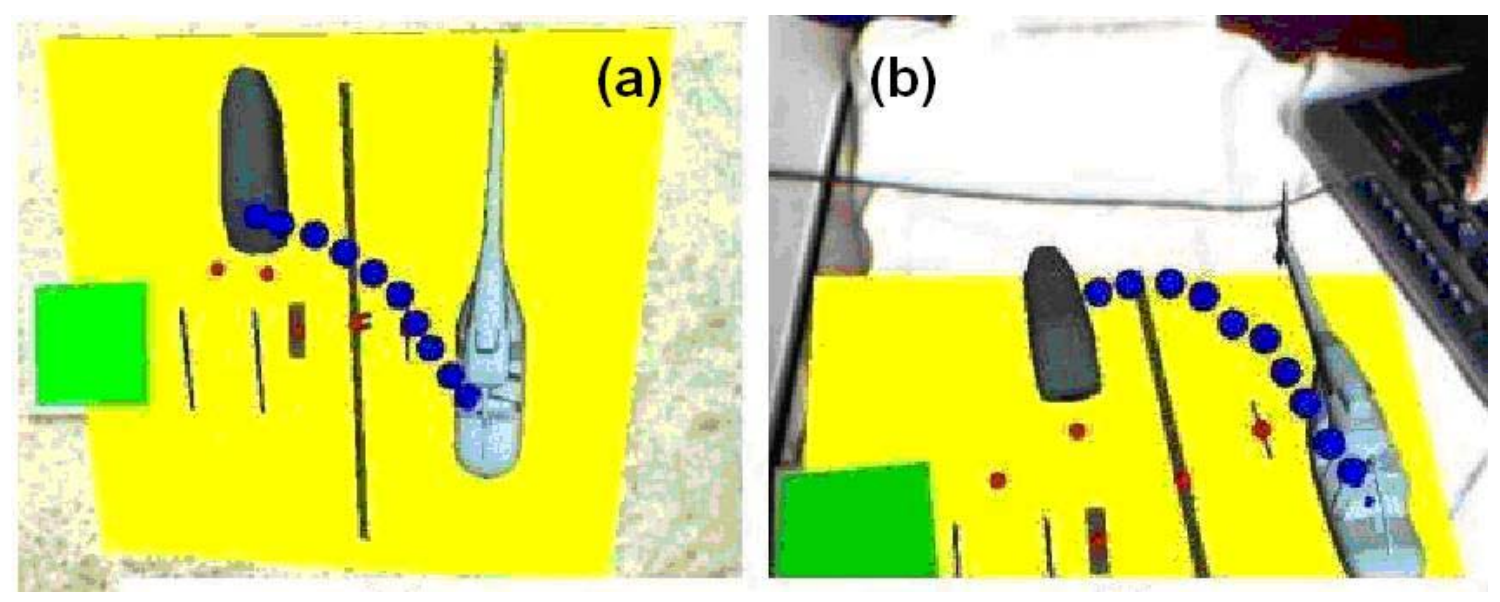

Figura 7 - Trajetória e colaboração remota no sistema SACRA; usuários remotos 1 (a) e 2 (b) (Santin, 2008)

\section{Conclusões}

A colaboração com o uso do computador, em processos pedagógicos de ensino/aprendizagem, vem crescendo e ganhando sofisticação como um elemento importante no relacionamento entre os indivíduos de um grupo. Novas tecnologias, como a Realidade Aumentada, estão permitindo replicar e ampliar as condições de colaboração local, uma vez que propiciam interações e comunicação tangíveis (com o uso das mãos), mais intuitivas e naturais, envolvendo objetos reais e virtuais 
misturados, complementados com elementos gráficos e sonoros. Nesse sentido, foram desenvolvidas algumas ferramentas de autoria, como é o caso dos projetos Construct3D e o sistema SACRA (projeto desenvolvido no Brasil).

O sistema SACRA mostrou-se uma poderosa ferramenta, por apresentar vários elementos favoráveis à colaboração avançada, como: interação tangível; criação e manipulação de pontos no espaço, associados a listas de objetos virtuais sonorizados com possibilidade de visualização de trajetórias; facilidade de autoria, sem a necessidade de letramento digital (bastam conhecimentos básicos de informática ao usuário para utilizar o SACRA no desenvolvimento de aplicações finais); atuação em aplicações colaborativas locais e em aplicações colaborativas remotas (à distância) síncronas e assíncronas. Enquanto as aplicações síncronas permitem a colaboração de grupos locais com grupos remotos em tempos real, as aplicações assíncronas podem ser feitas em tempos diferentes, assemelhando-se a um WIKI 3D, ou seja, um espaço colaborativo 3D pode ser usado ao longo do tempo, por várias pessoas, colaborando na montagem de cenários virtuais misturados com cenários reais.

Alguns testes do SACRA indicaram que ele pode ter muitos usos, permitindo atividades de treinamento, aprendizagem e entretenimento. Montagem de dispositivos, laboratórios virtuais e jogos são exemplos de aplicação do sistema SACRA.

A evolução do sistema SACRA deverá incorporar trechos de programa capazes de dar inteligência a objetos virtuais, permitindo o desenvolvimento de aplicações de hiper-realidade, incorporando objetos reais e virtuais inteligentes atuando em conjunto com os usuários.

\section{Referências Bibliográficas}

Agrawala, M. et al. The two-user responsive workbench: Support for collaboration through individual views of a shared space. In: Computer Graphics Proceedings. Los Angeles, 1997, Annual Conference Series, ACM SIGGRAPH, P. 327-332.

ARToolKit. Software Free e Open Source. Disponível em: $<$ http://www.hitl.washington.edu/artoolkit/> Acesso em 01.11.2008.

Azuma, R. et al. A Survey of Augmented Reality. Hughes Research Laboratories, Malibu, CA, 1997.

Bajura, M.; Neumann, U. (1995) "Dynamic Registration Correction in Video-Based Augmented Reality Systems" IEEE Computer Graphics \& Applications, v.15, n.5. p.52-60.

Barakonyi, I et al. (2003) "Augmented Reality Videoconferencing for Collaborative Work.", Proc. of the 2nd Hungarian Conference on Computer Graphics and Geometery. University of Technology, Budapest.

Billinghurst, M.; KATO, H. 2002. Collaborative augmented reality. Commun. ACM 45, 7, jul. 2002, P. 64-70.

Istituto Universal Brasileiro. <http://www.institutouniversal.com.br/historia.asp $>$ Acesso em 01.11.2008.

Kirner, C.; ZORZAL, E. R. Aplicações Educacionais em Ambientes Colaborativos com Realidade Aumentada. In: XVI Simpósio Brasileiro sobre Informática na Educação, 2005, Juiz de Fora - MG. Anais do XVI Simpósio Brasileiro de Informática na 
Educação. Porto Alegre - RS : Sociedade Brasileira de Computação - SBC, 2005. v. 1. p. 114-124.

Oliveira, L. A. Desenvolvimento do NetARToolKit: Um Sistema Distribuído de Realidade Aumentada. 119 p. Dissertação (Mestrado em Ciência da Computação). Universidade Metodista de Piracicaba, Piracicaba. 2008.

Santin, R. Sistema de Autotoria em Ambiente Colaborativo com Realidade Aumentada. 125 p. Dissertação (Mestrado em Ciência da Computação). Universidade Metodista de Piracicaba, Piracicaba. 2008.

Schmalstieg, D. et al. The Studierstube Augmented Reality Project PRESENCE - Teleoperators and Virtual Environments, Vol. 11, No. 1, pp. 32-54, MIT Press, February 2002.

Smith, B. L.; MacGregor, J. T. (1992). "What is collaborative learning?" In Goodsell, A. S., Maher, M. R., and Tinto, V. (Eds.), Collaborative Learning: A Sourcebook for Higher Education. National Center on Postsecondary Teaching, Learning, \& Assessment, Syracuse University.

Vivaty Studio. Software disponível em <http://www.vivaty.com/downloads/studio/ $>$ Acesso em 01.11.2008.

Zhou, Z. et al. (2004) "Interative Entertainment Systems Using Tangible Cubes", Australian Workshop on Interative Entertainment, p. 19-22. 